\title{
Osteopontin induces vascular endothelial growth factor expression in articular cartilage through PI3K/AKT and ERK1/2 signaling
}

\author{
JUN XU ${ }^{1}, \mathrm{YU} \mathrm{YI}^{1}, \mathrm{LIN} \mathrm{LI}^{2}$, WEIGUO ZHANG ${ }^{1}$ and $\mathrm{JIANHUA} \mathrm{WANG}^{3}$ \\ Departments of ${ }^{1}$ Orthopedic Surgery and ${ }^{2}$ Neurology, Wuhan Central Hospital, \\ Huazhong University of Science and Technology, Wuhan, Hubei 430030; ${ }^{3}$ Department of Orthopedic Surgery, Xinhua Hospital, \\ Shanghai Jiao Tong University School of Medicine, Shanghai 200092, P.R. China
}

Received August 27, 2014; Accepted May 8, 2015

DOI: $10.3892 / \mathrm{mmr} .2015 .3975$

\begin{abstract}
The expression of osteopontin (OPN) and vascular endothelial growth factor (VEGF) are associated with the severity of cartilage destruction in osteoarthritis. However, the biological connection between OPN and VEGF in osteoarthritis remains to be elucidated. The present study was performed to investigate the effect of OPN on VEGF expression in articular cartilage. Rat articular chondrocytes were isolated and cultured in monolayer conditions, and they were treated with OPN for different time periods $(0,2$, 8,12 or $24 \mathrm{~h}$ ) and dosages $(0,0.1,0.25$ or $0.5 \mu \mathrm{M})$. VEGF expression was assessed by reverse transcription-quantitative polymerase chain reaction and western blotting. The activation of the phosphoinositide 3-kinase (PI3K)/AKT and extracellular signal-regulated kinase (ERK)1/2 pathway was analyzed by detecting the expression of pPI3K, pAKT and pERK1/2. To inhibit the PI3K/AKT and ERK1/2 pathway, LY294002 and PD98059 were used, respectively or in combination. It was identified that OPN increased the expression of VEGF in a dose- and time-dependent manner. The PI3K/AKT and ERK1/2 pathways were activated following OPN stimulation and the effect was concomitant with the upregulation of VEGF. Finally, the regulation of VEGF was inhibited by LY294002 and PD98059, and their combination exhibited a synergistic effect. In conclusion, these findings suggest that OPN may
\end{abstract}

Correspondence to: Professor Jianhua Wang, Department of Orthopedic Surgery, Xinhua Hospital, Shanghai Jiao Tong University School of Medicine, 1665 Kongjiang Road, Shanghai 200092, P.R. China

E-mail shwangjianhua@gmail.com

Dr Jun Xu, Department of Orthopedic Surgery, Wuhan Central Hospital, Huazhong University of Science and Technology, 26 Shengli Street, Wuhan, Hubei 430030, P.R. China

E-mail: xujun7272@126.com

Key words: osteoarthritis, articular cartilage, osteopontin, angiogenesis, extracellular signal-regulated kinase/2 pathway, phosphoinositide 3-kinase/AKT pathway, vascular endothelial growth factor directly upregulate VEGF expression through the PI3K/AKT and ERK1/2 pathway. Further studies are required to reveal the mechanism of action of OPN on cartilage angiogenesis and cartilage destruction.

\section{Introduction}

Osteoarthritis (OA), the most common multifactorial degenerative joint disease in the elderly, is characterized by progressive degeneration of the articular cartilage, changes in the subchondral bone, osteophyte formation and synovial inflammation (1). The etiology of OA involves numerous mechanical and biochemical factors (2-5).

Healthy articular cartilage is essentially avascular and is resistant to vascular invasion in vitro $(6,7)$. In OA, however, the invasion of blood vessels from the subchondral bone is apparent even in the early stages of the disease and subsequently leads to the loss of tidemark integrity (8-11). Angiogenesis is dependent on a complex network, which is regulated in a timely and sequential order to mediate blood vessel formation, and vascular endothelial growth factor (VEGF) has been recognized as a dominant mediator of this process (12). VEGF-dependent signaling in embryonic development is important for the regulation of growth plate morphogenesis and the coupling between cartilage and bone formation (13). As healthy adult cartilage is essentially avascular, VEGF is not expressed in normal chondrocytes. However, several studies have revealed the expression of VEGF and the corresponding receptors in OA (14-17).

Osteopontin (OPN) is a member of the small integrin-binding ligand $\mathrm{N}$-linked glycosylated protein family. It is abundant in the extracellular matrix of mineralized tissues, such as bone, where it mediates important cell-matrix and cell-cell interactions $(18,19)$. Upregulation of OPN has been observed in human cartilage from patients with OA, and plasma and synovial fluid OPN levels were increased in patients with primary knee OA, which were shown to be correlated with more severe OA $(20,21)$. OPN may be thus involved in the molecular pathogenesis of OA, contributing to the progressive degeneration of articular cartilage. OPN is hypothesized to be involved in the destruction of the cartilage matrix by inducing the production of matrix metalloproteinases (MMPs) in articular chondrocytes (22). 
The aim of the present study was to investigate the effect of OPN on VEGF levels in articular cartilage cells, and evaluate the possible underlying mechanisms involved.

\section{Materials and methods}

Cell culture and treatment. A total of 12 Sprague-Dawley (SD) rats (age, 1 week) were purchased from Wuhan University Center for Animal Experiment (Wuhan, China). The study was approved by the institutional review board of Wuhan Central Hospital, and all procedures complied with the Guide for the Care and Use of Laboratory Animals (National Institutes of Health, Bethesda, MD, USA). Briefly, articular chondrocytes were isolated from the knee joint of SD rats, cartilage tissues were obtained and cut into small sections as previously described (23). The cartilage slices were further dissociated enzymatically for $2 \mathrm{~h}$ with $0.2 \%$ type II collagenase (Sigma-Aldrich, St. Louis, MO, USA) at $37^{\circ} \mathrm{C}$. The cells were collected and resuspended in culture medium (Dulbecco's modified Eagle's medium/F12 supplemented with $10 \%$ fetal bovine serum, $100 \mathrm{U} / \mathrm{ml}$ penicillin and $100 \mu \mathrm{g} / \mathrm{ml}$ streptomycin; Invitrogen Life Technologies, Carlsbad, CA, USA) and cultured in a $37^{\circ} \mathrm{C}$, humidified, $5 \% \mathrm{CO}_{2}$ incubator. The medium was replaced every other day until the cells reached $80 \%$ confluence. The chondrocytes were confirmed by aggrecan and collagen-II expression. The expression of aggrecan and collagen-II were examined by immunohistochemistry. The chondrocytes were confirmed by positive expression of aggrecan and collagen-II. The cell viability was assessed using trypan blue staining (Sigma-Aldrich). Cells were exposed to OPN for different durations $(0,2,8$, 12 or $24 \mathrm{~h})$ and dosages $(0,0.1,0.25$ or $0.5 \mu \mathrm{M})$. To inhibit the phosphoinositide 3-kinase (PI3K)/AKT and extracellular signal-regulated kinase (ERK)1/2 pathways, LY294002 $(10 \mu \mathrm{M})$ and PD98059 $(20 \mu \mathrm{M})$ were used respectively or in combination. Each experiment was repeated at least 3 times.

RNA isolation and reverse transcription-quantitative polymerase chain reaction $(R T-q P C R)$. The total RNA was extracted from cells using the RNeasy plus mini kit (Qiagen China Co., Ltd, Shanghai, China) and reverse-transcribed into cDNA according to the manufacturer's instructions. RT-qPCR was performed using the Applied Biosystems 7500 Real-time PCR system (Applied Biosystems, Foster City, CA, USA), and the SYBR Green fluorescent dye (Invitrogen Life Technologies) method was used to quantify the cDNA. $\beta$-actin was used as the internal control. The relative contents of the copy numbers of the target gene mRNA were then calculated using the $2^{-\Delta \Delta C t}$ method. All experiments were performed in triplicate. The primers sequences were as follows: VEGF, forward 5'-TGT GAA TGC AGA CCA AAG AAA GA-3' and reverse 5'-GCT TTC TCC GCT CTG AGC AA-3'; $\beta$-actin, forward 5'-GTC CAC CGC AAA TGC TTC TA-3' and reverse 5'-TGC TGT CAC CTT CAC CGT TC-3'. Target sequences were amplified at $95^{\circ} \mathrm{C}$ for $10 \mathrm{~min}$, followed by 40 cycles of $95^{\circ} \mathrm{C}$ for $15 \mathrm{sec}$ and $60^{\circ} \mathrm{C}$ for $1 \mathrm{~min}$.

Western blotting. Cell lysates were prepared using cell lysis radioimmunoprecipitation assay buffer (Sigma-Aldrich). The protein concentrations were determined using the bicinchoninic acid protein assay kit (Sigma-Aldrich). For each sample, a total of $40 \mu \mathrm{g}$ protein was separated using $10 \%$ SDS-PAGE. The separated proteins were then transferred onto a polyvinylidene difluoride membrane (Life Technologies, Carlsbad, CA, USA). Following blocking with 5\% non-fat milk in Tris-buffered saline containing $0.05 \%$ Tween-20 (TBST; Life Technologies), the membrane was incubated with the following primary antibodies: Rabbit polyclonal anti-human VEGF (1:1,000; cat. no. ab46154; Abcam, Cambridge, MA, USA), mouse monoclonal anti-human PI3K and rabbit polyclonal anti-human phospho-PI3K (1:500; cat. nos. ab182651 and ab189403; Abcam), Rabbit polyclonal anti-human pan-AKT and phosphor-AKT1 (1:1,000; cat. no. ab8805 and ab66138; Abcam) and mouse monoclonal anti-human $\beta$-actin $(1: 2,000$; cat. no. sc-47778; Santa Cruz Biotechnology, Inc., Santa Cruz, CA, USA) in TBST at $4^{\circ} \mathrm{C}$ overnight. The membrane was then washed three times with TBST and incubated with the horseradish peroxidase-conjugated secondary antibody $(1: 3,000$; Santa Cruz Biotechnology, Inc.) in TBST for $2 \mathrm{~h}$ at room temperature. The membrane was washed again, followed by visualization using an enhanced chemiluminescence substrate (Sigma-Aldrich). The densitometric data were obtained using Image $\mathbf{J}$ software (National Institutes of Health).

Statistical analysis. All data are presented as the mean \pm standard deviation. Statistical analysis was performed using SPSS version 13.0 software (SPSS, Inc., Chicago, IL, USA). A one-way analysis of variance was performed. $\mathrm{P}<0.05$ was considered to indicate a statistically significant difference.

\section{Results}

$O P N$ increases the expression of VEGF in a dose-and time-dependent manner. To investigate whether OPN regulates the expression of VEGF in chondrocytes, the expression of VEGF was analyzed initially in chondrocytes treated with OPN at different concentrations and time-durations. As shown in Fig. 1A, OPN increased VEGF mRNA expression in a dose-dependent manner. Incubation of chondrocytes with $0.25 \mu \mathrm{M}$ OPN resulted in a statistically significant increase in the relative level of VEGF, while incubation with $0.5 \mu \mathrm{M}$ OPN resulted in an almost 4-fold increase. It was then established whether there was a time-dependent regulation and chondrocytes were incubated with $0.5 \mu \mathrm{M}$ OPN. It was found that VEGF mRNA levels were increased substantially by $4 \mathrm{~h}$ and relatively high levels persisted for $24 \mathrm{~h}$ after stimulation with OPN (Fig. 1B). In addition, VEGF expression was assessed using western blot analysis. The protein level was consistent with the mRNA level of VEGF, which also exhibited a dose- and time-dependent regulation by OPN (Fig. 1C and D).

PI3K/AKT and ERK1/2 pathways are activated by OPN. To investigate the signaling pathway involved, the phosphorylation of PI3K/AKT and ERK1/2 was assessed. PI3K/AKT activation was observed following exposure to $0.25 \mu \mathrm{M}$ and $0.5 \mu \mathrm{M}$ OPN, in a dose-dependent manner (Fig. 2A). The OPN-induced phosphorylation of PI3K/AKT was also found following exposure to OPN for $4 \mathrm{~h}$, and lasted for $24 \mathrm{~h}$ (Fig. 2B). A concentration-dependent effect of OPN on chondrocytes causing ERK1/2 phosphorylation was also observed 
A

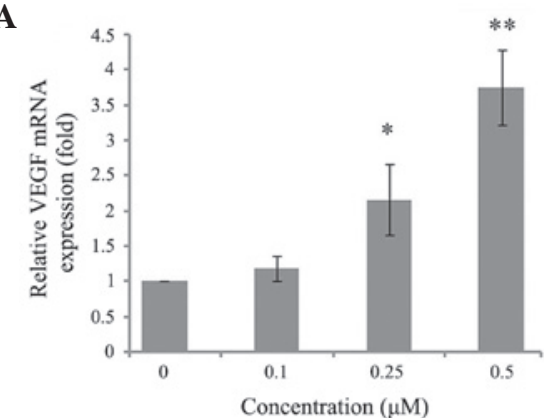

C

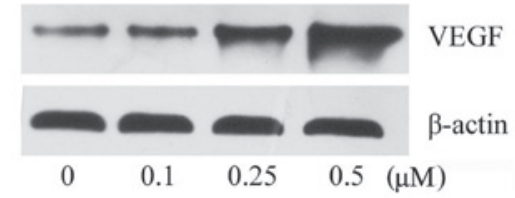

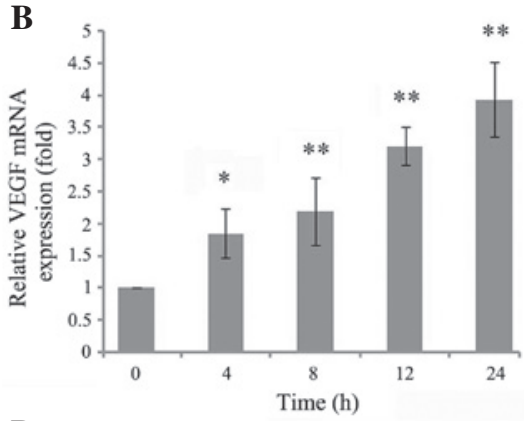

D

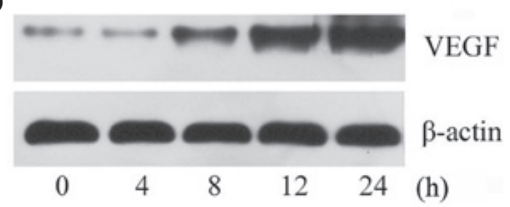

Figure 1. OPN induces the expression of VEGF in a dose- and time-dependent manner. Chondrocytes were cultured in the presence of OPN at various concentrations and for certain time periods. (A) Levels of VEGF mRNA were determined by reverse transcription-quantitative polymerase chain reaction. OPN increased the level of VEGF mRNA in a dose-dependent manner. (B) OPN increased the level of VEGF mRNA in a time-dependent manner. (C and D) The expression of VEGF was detected using western blotting, and OPN increased the level of VEGF protein in a dose- and time-dependent manner. OPN, osteopontin; VEGF, vascular endothelial growth factor.

A

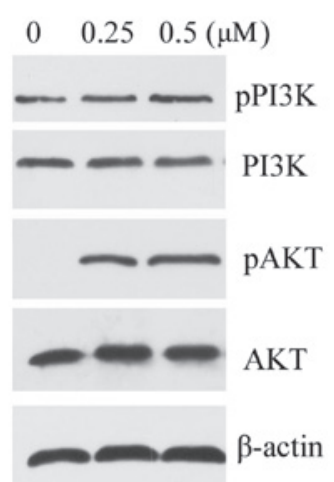

C

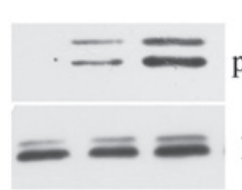

B $\begin{array}{llllll}0 & 2 & 4 & 8 & 24 & (\mathrm{~h})\end{array}$

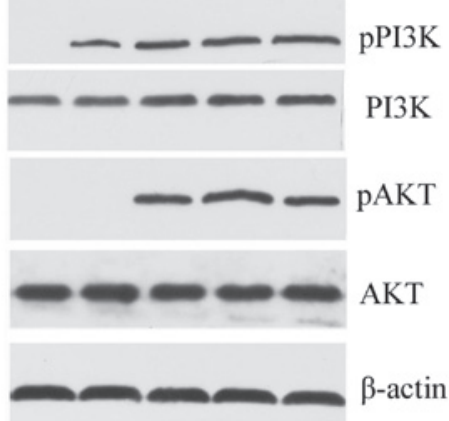

D

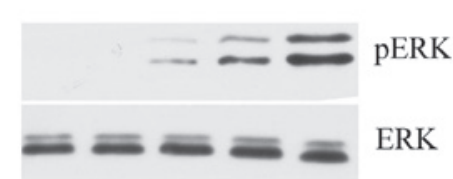

Figure 2. OPN induces the phosphorylation of PI3K/AKT and ERK1/2 in a dose- and time-dependent manner. Chondrocytes were cultured in the presence of OPN at various concentrations and time periods. The phosphorylation of PI3K/AKT and ERK1/2 was determined by western blotting. (A and B) OPN induced the phosphorylation of PI3K/AKT in a dose- and time-dependent manner. (C and D) OPN induced the phosphorylation of ERK in a dose- and time-dependent manner. OPN, osteopontin; PI3K, phosphoinositide 3-kinase; ERK, extracellular signal-regulated kinase.

A

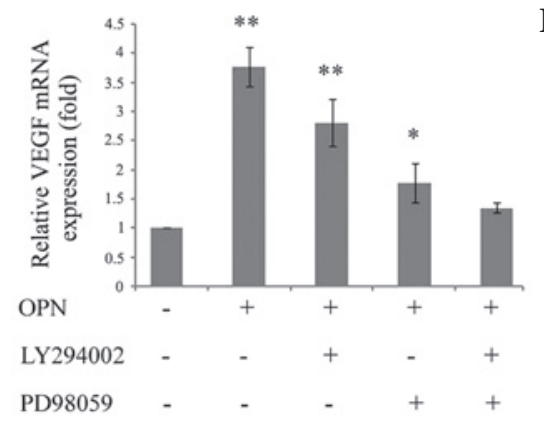

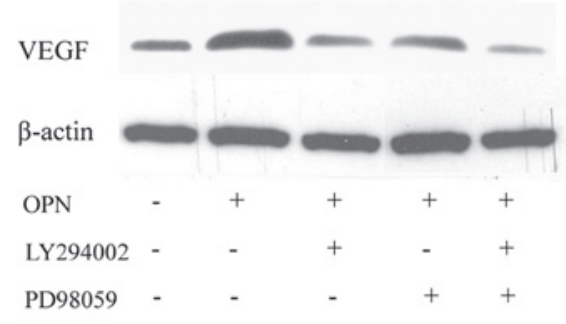

Figure 3. OPN induction of VEGF production is blocked by PI3K/AKT and ERK1/2 pathway inhibition. (A) Levels of VEGF mRNA were determined by reverse transcription-quantitative polymerase chain reaction, OPN-induced VEGF mRNA was significantly decreased by either LY294002 or PD98059, and the two inhibitors exhibited a synergistic effect. (B) Levels of VEGF protein were determined by western blotting, the protein level was consistent with the mRNA level of VEGF, which also demonstrated a PI3K/AKT- and ERK1/2-dependent regulation by OPN. The combination of the two inhibitors could completely reverse the effect. OPN, osteopontin; VEGF, vascular endothelial growth factor; PI3K, phosphoinositide 3-kinase; ERK, extracellular signal-regulated kinase. 
(Fig. 2C). The time course for the phosphorylation of ERK1/2 was almost the same as for PI3K (Fig. 2D).

Blocking PI3K/AKT and ERK1/2 pathways inhibits VEGF production. To determine whether OPN induced VEGF expression through the PI3K/AKT and ERK1/2 signaling pathways, the cells were treated with LY294002 and PD98059. It was identified that OPN-induced VEGF mRNA was significantly inhibited by either LY294002 or PD98059. While a single pathway inhibitor may partially reduce VEGF mRNA expression, the combination of the two inhibitors exhibited a synergistic effect and was able to completely reverse the increase (Fig. 3A). These data indicated that the PI3K/AKT and ERK1/2 signaling pathways may have an important function in OPN-induced VEGF expression in chondrocytes. In addition, VEGF expression was assessed using western blotting. The protein level was consistent with the mRNA level of VEGF, which also exhibited PI3K/AKT- and ERK1/2-dependent regulation by OPN (Fig. 3B).

\section{Discussion}

In pathological conditions, such as OA, damaged articular cartilage is frequently covered with and invaded by granulation tissue with a high level of vascularization. These observations in the pathophysiological conditions suggest the involvement of angiogenic factors in the process of OA $(24,25)$.

OPN has become a focus in OA pathogenesis research in previous decades. Previous studies have revealed a close association between OPN and OA $(20,21)$. Recent studies have also revealed that VEGF is important in the degenerative process of OA $(13,14,17)$. VEGF has marked angiogenic activity with specific mitogenic and chemotactic actions on endothelial cells. In the present study, the data indicated that OPN enhanced the expression of VEGF in chondrocytes. A previous study revealed that OPN promotes the expression of MMP13 (22), while VEGF inhibited the expression of aggrecan and type II collagen (26). OPN and VEGF may destroy the framework of articular cartilage. This process of extracellular matrix degradation is required for angiogenesis and therefore accelerates the angiogenic function of VEGF in cartilage.

To the best of our knowledge, this is the first study in which OPN has been associated with VEGF in OA. The signaling pathway that may connect OPN and VEGF was further investigated. The signaling pathways involved in the function of OPN appear to vary among different cell types. For instance, the PI3K pathway has been observed to be induced by OPN in breast cancer cells (27), while the nuclear factor- $\kappa \mathrm{B}$ signaling cascade was involved in OPN-induced tumor cell migration and epithelial cell motility in prostate cancer (28). In lymphocytes, the mitogen-activated protein kinase/ERK-mediated signaling pathway was associated with OPN-induced migration and motility (29). In the present study, the PI3K/AKT and ERK1/2 signaling pathways were activated following administration of OPN and persisted for at least $24 \mathrm{~h}$, and OPN-induced phosphorylation occured in a dose-dependent manner. These findings suggested that the signaling cascades may be associated with OPN-induced VEGF expression. In addition, when blocking the activation of these two pathways using a PI3K inhibitor and ERK inhibitor, OPN-induced VEGF expression was significantly decreased. While a single pathway inhibitor partially reduced VEGF mRNA expression, the combination of the two inhibitors completely reversed the effect. These data indicated that OPN enhanced the expression of VEGF through phosphorylation of PI3K/AKT and ERK1/2.

In conclusion, the present results revealed an association between OPN and VEGF in articular cartilage. It was observed that OPN treatment resulted in an elevated expression of VEGF at the gene level and protein level, and the effect was induced through activation of the PI3K/AKT and ERK1/2 signaling pathways. Additional studies are required to reveal the mechanism of action of OPN in cartilage angiogenesis and cartilage destruction.

\section{Acknowledgements}

The present study was supported by the National Natural Science Foundation of China (grant no. 81171706) and the Shanghai Municipal Natural Science Foundation (grant no. 11ZR1427400).

\section{References}

1. Gonzalez A: Osteoarthritis year 2013 in review: Genetics and genomics. Osteoarthritis Cartilage 21: 1443-1451, 2013.

2. Barg A, Pagenstert GI, Hugle T, Gloyer M, Wiewiorski M, Henninger HB and Valderrabano V: Ankle osteoarthritis: Etiology, diagnostics and classification. Foot Ankle Clin 18: 411-426, 2013.

3. Sandell LJ: Etiology of osteoarthritis: Genetics and synovial joint development. Nat Rev Rheumatol 8: 77-89, 2012.

4. Soder S and Aigner T: Osteoarthritis. Etiology,typing, staging and histological grading. Pathologe 32: 183-192, 2011 (In German).

5. Michael JW, Schluter-Brust KU and Eysel P: The epidemiology, etiology, diagnosis and treatment of osteoarthritis of the knee. Dtsch Arztebl Int 107: 152-162, 2010.

6. Bara JJ, Johnson WE, Caterson B and Roberts S: Articular cartilage glycosaminoglycans inhibit the adhesion of endothelial cells. Connect Tissue Res 53: 220-228, 2012.

7. Hyc A, Osiecka-Iwan A, Jóźwiak J and Moskalewski S: The morphology and selected biological properties of articular cartilage. Ortop Traumatol Rehabil 3: 151-162, 2001.

8. Ashraf S, Mapp PI and Walsh DA: Contributions of angiogenesis to inflammation, joint damage and pain in a rat model of osteoarthritis. Arthritis Rheum 63: 2700-2710, 2011.

9. Pesesse L, Sanchez C and Henrotin Y: Osteochondral plate angiogenesis: A new treatment target in osteoarthritis. Joint Bone Spine 78: 144-149, 2011.

10. Mapp PI and Walsh DA: Mechanisms and targets of angiogenesis and nerve growth in osteoarthritis. Nat Rev Rheumatol 8: 390-398, 2012.

11. Wang QY, Dai J, Kuang B, Zhang J, Yu SB, Duan YZ and Wang MQ: Osteochondral angiogenesis in rat mandibular condyles with osteoarthritis-like changes. Arch Oral Biol 57: 620-629, 2012.

12. Nowak DG, Amin EM, Rennel ES, Hoareau-Aveilla C, Gammons M, Damodoran G, Hagiwara M, Harper SJ, Woolard J, Ladomery MR, et al: Regulation of vascular endothelial growth factor (VEGF) splicing from pro-angiogenic to anti-angiogenic isoforms: A novel therapeutic strategy for angiogenesis. J Biol Chem 285: 5532-5540, 2010.

13. Lingaraj K, Poh CK and Wang W: Vascular endothelial growth factor (VEGF) is expressed during articular cartilage growth and re-expressed in osteoarthritis. Ann Acad Med Singapore 39: 399-403, 2010.

14. Jansen H, Meffert RH, Birkenfeld F, Petersen W and Pufe T: Detection of vascular endothelial growth factor (VEGF) in moderate osteoarthritis in a rabbit model. Ann Anat 194: 452-456, 2012.

15. Yamairi F, Utsumi H, Ono Y, Komorita N, Tanaka M and Fukunari A: Expression of vascular endothelial growth factor (VEGF) associated with histopathological changes in rodent models of osteoarthritis. J Toxicol Pathol 24: 137-142, 2011. 
16. Murata M, Yudoh $\mathrm{K}$ and Masuko K: The potential role of vascular endothelial growth factor (VEGF) in cartilage: How the angiogenic factor could be involved in the pathogenesis of osteoarthritis? Osteoarthritis Cartilage 16: 279-286, 2008.

17. Matsumoto T, Cooper GM, Gharaibeh B, Meszaros LB, Li G, Usas A, Fu FH and Huard J: Blocking VEGF as a potential approach to improve cartilage healing after osteoarthritis J Musculoskelet Neuronal Interact 8: 316-317, 2008.

18. Rangaswami H, Bulbule A and Kundu GC: Osteopontin: Role in cell signaling and cancer progression. Trends Cell Biol 16: 79-87, 2006.

19. Chakraborty G, Jain S, Behera R, Ahmed M, Sharma P, Kumar V and Kundu GC: The multifaceted roles of osteopontin in cell signaling, tumor progression and angiogenesis. Curr Mol Med 6: 819-830, 2006

20. Gao SG, Li KH, Zeng KB, Tu M, Xu M and Lei GH: Elevated osteopontin level of synovial fluid and articular cartilage is associated with disease severity in knee osteoarthritis patients. Osteoarthritis Cartilage 18: 82-87, 2010.

21. Honsawek S, Tanavalee A, Sakdinakiattikoon M Chayanupatkul M and Yuktanandana P: Correlation of plasma and synovial fluid osteopontin with disease severity in knee osteoarthritis. Clin Biochem 42: 808-812, 2009.

22. Xu M, Zhang L, Zhao L, Gao S, Han R, Su D and Lei G: Phosphorylation of osteopontin in osteoarthritis degenerative cartilage and its effect on matrix metalloprotease 13. Rheumatol Int 33: 1313-1319, 2013.
23. Chen Q, Liu SQ, Du YM, Peng $H$ and Sun LP: Carboxymethyl-chitosan protects rabbit chondrocytes from interleukin-1beta-induced apoptosis. Eur J Pharmacol 541: $1-8,2006$

24. Ashraf S and Walsh DA: Angiogenesis in osteoarthritis. Curr Opin Rheumatol 20: 573-580, 2008

25. Mapp PI, Avery PS, McWilliams DF, Bowyer J, Day C, Moores S, Webster R and Walsh DA: Angiogenesis in two animal models of osteoarthritis. Osteoarthritis Cartilage 16: 61-69, 2008.

26. Chen XY, Hao YR, Wang Z, Zhou JL, Jia QX and Qiu B: The effect of vascular endothelial growth factor on aggrecan and type II collagen expression in rat articular chondrocytes. Rheumatol Int 32: 3359-3364, 2012.

27. Das R, Mahabeleshwar GH and Kundu GC: Osteopontin stimulates cell motility and nuclear factor kappaB-mediated secretion of urokinase type plasminogen activator through phosphatidylinositol 3-kinase/Akt signaling pathways in breast cancer cells. J Biol Chem 278: 28593-28606, 2003.

28. Jain S, Chakraborty G and Kundu GC: The crucial role of cyclooxygenase-2 in osteopontin-induced protein kinase $\mathrm{C}$ alpha/c-Src/IkappaB kinase alpha/beta-dependent prostate tumor progression and angiogenesis. Cancer Res 66: 6638-6648, 2006.

29. Cao Z, Dai J, Fan K, Wang H, Ji G, Li B, Zhang D, Hou S, Qian W, Zhao J, et al: A novel functional motif of osteopontin for human lymphocyte migration and survival. Mol Immunol 45: 3683-3692, 2008. 